\title{
Esofagite eosinofílica em pacientes pediátricos: uma revisão de literatura
}

\author{
Eosinophilic esophageal in pediatric patients: a literature review
}

Esofagite eosinofilica en pacientes pediatricos: una revisión de literatura

Dandara Freitas da Silveira e Souza ${ }^{1 *}$, Igor Machado Von Anchen Erdmann ${ }^{*}$, Thaís Bichiatto" Thais Martins Oliveira Santos ${ }^{1}$.

\section{RESUMO}

Objetivo: Os autores buscaram revisar na literatura a fisiopatologia, quadro clínico e tratamento da esofagite eosinofílica em pacientes pediátricos. Métodos: Trata-se de um estudo observacional, em que se realizou revisão literária de 53 artigos, abrangendo os estudos publicados nos últimos 10 anos. Resultados: $\mathrm{A}$ esofagite eosinofílica é uma doença esofágica inflamatória crônica, histologicamente caracterizada por infiltração de eosinófilos isolados na mucosa do esôfago. Patogênese comum em crianças com contribuição importante de fatores genéticos e ambientais. O diagnóstico é feito através da endoscopia digestiva alta (EDA), baseado na histologia das biópsias coletadas. Novas diretrizes têm defendido a eficácia de tratamentos com inibidores de bomba de prótons (IBP), corticoterapia e dietas de restrição alimentar, apresentando boa resposta clínica e histológica. Considerações finais: A melhor compreensão do quadro clínico, diagnóstico e tratamento permitiram melhorar o prognóstico das crianças portadoras desta patologia subdiagnosticada atualmente.

Palavras-chave: Esofagite eosinofílica, Endoscopia digestiva alta, Eosinófilos.

\begin{abstract}
Objective: The authors sought to review in the literature the pathophysiology, clinical status and treatment of eosinophilic esophagitis in pediatric patients. Methods: This is an observational study, in which a literary review of 53 articles was carried out, covering the studies published in the last 10 years. Results: Eosinophilic esophagitis is a chronic inflammatory esophageal disease, histologically characterized by infiltration of isolated eosinophils in the mucosa of the esophagus. Common pathogenesis in children with important contribution of genetic and environmental factors. The diagnosis is made through upper digestive endoscopy (EDA), based on the histology of the biopsies collected. New guidelines have defended the effectiveness of treatments with proton pump inhibitors (PPIs), corticosteroids and dietary restriction diets, presenting good clinical and histological response. Conclusion: A better understanding of the clinical picture, diagnosis and treatment allowed to improve the prognosis of the children with this current underdiagnosed condition.
\end{abstract}

Keywords: Eosinophilic esophagitis, Upper digestive endoscopy, Eosinophils.

\section{RESUMEN}

Objetivo: Los autores buscaron revisar en la literatura la fisiopatología, cuadro clínico y tratamiento de la esofagitis eosinofílica en pacientes pediátricos. Métodos: Se trata de un estudio observacional, en que se realizó una revisión literaria de 53 artículos, abarcando los estudios publicados en los últimos 10 años. Resultados: La esofagitis eosinofílica es una enfermedad esofágica inflamatoria crónica, histológicamente

${ }^{1}$ Universidade de Franca (UNIFRAN), Franca, São Paulo, Brasil. *E-mail: igorerdmann17@gmail.com 
caracterizada por infiltración de eosinófilos aislados en la mucosa del esófago. Patogénesis común en niños con una contribución importante de factores genéticos y ambientales. El diagnóstico se realiza a través de la endoscopia digestiva alta (EDA), basado en la histología de las biopsias recogidas. Las nuevas directrices han defendido la eficacia de tratamientos con inhibidores de bomba de protones (IBP), corticoterapia y dietas de restricción alimentaria, presentando buena respuesta clínica e histológica. Conclusión: La mejor comprensión del cuadro clínico, diagnóstico y tratamiento permitieron mejorar el pronóstico de los niños portadores de esta patología subdiagnosticada actualmente.

Palabras clave: Esofagitis eosinofílica, Endoscopia digestiva alta, Eosinófilos.

\section{INTRODUÇÃO}

A esofagite eosinofílica (EoE), é uma condição clínico-patológica de curso crônico e de localização esofágica. Acomete principalmente meninos (proporção de 3:1), afetando a faixa etária pediátrica com predomínio entre os cinco e dez anos, sendo relatado mais em países desenvolvidos e em indivíduos caucasianos. Sua incidência varia de 5 a 10 casos por 1.000 .000 ao redor do mundo. A prevalência e incidência da mesma têm aumentado progressivamente uma vez que os profissionais de saúde estão se informando mais acerca da doença (LITOSH VA, et al. 2017).

Acredita-se que fatores como a genética e algumas exposições nos primeiros anos de vida, tais como alimentação exclusiva de fórmulas infantis ou mistas, antibioticoterapia precoce, parto prematuro e cesárea estejam relacionadas a essa maior ocorrência (LITOSH VA, et al. 2017).

Estudos envolvendo a genética molecular e o silenciamento de genes, explicam a interferência de enzimas como a "Calpain-14" (CAPN14) na qual sua expressão desregulada é induzida por interleucinas, principalmente interleucina-13 (IL-13) em células epiteliais esofágicas que compromete a barreira da região, modificando negativamente sua integridade, reduzindo desse modo a 1-desmogleína (Dsg1), glicoproteína que auxilia no combate a forças que promovem stress mecânico em tal epitélio (LITOSH VA, et al. 2017).

Notando assim que a EoE pode ser induzida por mutações expressadas pelo silenciamento de genes (WECHSLER JB e BRYCE PJ, 2014).

Com o aumento de IL-13, as células do epitélio esofágico passam a manifestar a eotaxina-3, que recruta eosinófilos para o esôfago. Esses produzem a IL-13 causando liberação de neurotoxina derivada de eosinófilos (EDN), que irão ativar células dendríticas para diferenciar os linfócitos $T$ em linfócitos $T$ auxiliar do tipo 2(Th2), e citocinas Th2, propagando inflamação (WECHSLER JB e BRYCE PJ, 2014).

Teoricamente, um tratamento baseado no bloqueio da Imunoglobulina $E(\lg E)$ iria induzir a erradicação da doença. Porém, segundo estudos, a utilização de anticorpos anti-lgE não foi eficaz para o tratamento da patologia. Com isto, foi concluído que há outros mecanismos não lgE mediados capazes de gerar a doença (WESCHSLER JB e BRYCE PJ, 2014).

O quadro clínico da EoE é semelhante ao da doença do refluxo gastroesofágico (DRGE), principalmente em lactentes e pré-escolares, manifestando com náuseas, vômitos, dor abdominal inespecífica, impactação alimentar e baixo ganho ponderal. Já em escolares e adolescentes, os sintomas apresentam dor abdominal, vômitos e impactação de alimentos sólidos (LYNCH MK, et al. 2015).

É frequente encontrar nesses pacientes quadros de atopia, como rinite alérgica, asma, eczema e alergia alimentar (ACEVES SS, 2014).

Para que seja concluído o diagnóstico, além das manifestações clínicas, tem como padrão ouro a realização de 4 a 6 biópsias do esôfago proximal e distal Durrani $S$ (2017), esclarecendo a variação histopatológica da mucosa como infiltrado eosinofílico e a inflamação, sendo estas adquiridas na endoscopia digestiva alta (EDA), pois a infiltração se manifesta de forma irregular, podendo não ser adequado para diagnóstico amostra recolhida de um único local (CIANFERONI A, et al. 2015). 
O prognóstico dos pacientes propende a persistir ou recorrer até a vida adulta, com períodos de remissão e recidiva. As complicações a longo prazo são surgimento de estenoses fibróticas consequente a cronicidade da inflamação e deposição de colágeno. Até então, não foram constatados na literatura casos de malignidade relacionados a patologia (HIRANO I e ACEVE SS, 2014).

Desta maneira, o objetivo desta revisão literária é conhecer a clínica apresentada pelos portadores de EoE, assim como diagnosticar precocemente a comorbidade e tratar de maneira adequada a fim de evitar possíveis complicações e prejuízos na qualidade de vida dos pacientes.

\section{METODOLOGIA}

Este estudo trata-se de uma revisão sistemática de literatura, realizado por estudantes de medicina da Universidade de Franca (UNIFRAN). A revisão literária iniciou-se em novembro de 2018 e teve sua finalização em abril de 2019, com buscas ativas por artigos publicados em fontes como: Biblioteca Nacional de Medicina dos EUA (PubMed), Centro Latino Americano e do Caribe de Informação em Ciência da Saúde (Bireme), Scientific Electronic Library Online e Portal de Periódicos (CAPES). Foram utilizados como descritores nas referências "Esofagite Eosinofílica Pediatria." Os critérios de inclusão nesse estudo foram artigos científicos publicados nos últimos 10 anos (2008 - 2018), sendo selecionados aqueles que apresentaram enfoque no tema esofagite esinofílica, elegidos por grande e pouca relevância. Durante o período do estudo, foram encontrados 53 artigos, destes, 16 literaturas foram descartadas por não estarem de acordo com os critérios de inclusão. No entanto, para realização deste trabalho, foram elegidos 21 referências com maior relevância no assunto.

\section{REVISÃO DE LITERATURA}

A esofagite eosinofílica (EoE), na faixa etária pediátrica, assume uma variedade de sintomas que devem ser diferenciados de outras patologias como a Doença do Refluxo Gastroesofágico (DRGE). O diagnóstico absoluto é com base na presença de pelo menos 15 eosinófilos/CGA encontrados na análise da biópsia endoscópica. As medidas terapêuticas preconizadas são as dietéticas e medicamentosas principalmente (BRAGA-TAVARES H, et al. 2009).

Diante do quadro clínico, as crianças menores costumam sentir sintomas inespecíficos como problemas de alimentação, vômitos/regurgitação, recusa alimentar, engasgos e dor abdominal. Já os adolescentes e adultos referem dor retroesternal, pirose, impactação alimentar e disfagia, podendo esses serem intermitentes ou um pouco mais contínuos, ou ainda, graves ou sutis dependendo, por exemplo, da consistência alimentar e da velocidade do trânsito alimentar (CARR S, 2018). No entanto, deve-se recordar que alguns pacientes que sofrem da EoE são assintomáticos (HIRANO I e ACEVES SS, 2014). As crianças muitas vezes fazem uso de artifícios como o uso de bebidas durante as refeições e de produtos como solventes que podem auxiliar na lubrificação do canal a fim de evitar engasgos alimentares, utilizando assim, de mecanismos compensatórios visando uma adequada adaptação alimentar (WECHSLER JB e BRYCE PJ, 2014).

Na maioria das vezes, o exame físico apresenta-se normal, exceto nas manifestações atópicas associadas como a eosinofilia periférica. Outro fator importante são os sintomas que não respondem a tratamentos médicos ou cirúrgicos para doença do refluxo gastresofágico (DRGE) (CHEHADE, 2016). Os pilares necessários para diagnosticar englobam suspeita clínica associados a achados endoscópicos e histológicos (BRAGA-TAVARES H, et al. 2009).

Não existe um sinal patognomônico, porém podemos encontrar alterações como estreitamento do calibre esofagiano; estenoses; edema e consequente apagamento da trama vascular; sulcos ou estrias verticais; anéis concêntricos de Schatski transitórios ou fixos, conferindo uma traquealização; exsudato granular e fragilidade da mucosa (MUIR AB, et al. 2016). Uma outra utilidade da endoscopia digestiva alta (EDA) proposta na Escala de Referência Endoscópica para EoE (EREFS) é a diferenciação de dois padrões endoscópicos. O inflamatório tem a presença de exsudato, anéis transitórios (felinização), estrias verticais e 
edema, sendo predominantemente encontrado na faixa pediátrica. No fibroestenótico, tem-se anéis fixos característicos da traquealização, estenoses, redução do calibre esofágico e esôfago em "papel crepom" devido sua mucosa fragilizada (ACEVES SS, 2014). De acordo com diversas literaturas, a condição histológica é caracterizada pela coleta de biópsias, sendo pelo menos 4 a 6 fragmentos cobrindo os três terços da mucosa esofágica, até mesmo áreas visualmente normais (DURRANI S, 2017). O diagnóstico absoluto é com base na presença de pelo menos 15 eosinófilos/CGA (x400) mesmo após a intervenção medicamentosa de antiácidos com elevadas doses de Inibidor da Bomba de Prótons (IBP) (ROTHENBERG ME, 2015).

Deve-se salientar que os achados histológicos, tal como a contagem de eosinófilos, são significativos para qualificar a resposta do paciente mediante a administração do tratamento (ROTHENBERG ME, 2015). O esofagograma contrastado com bário pode ser usado para evidenciar essas alterações em indivíduos com sintomas evocativos a EoE, sendo esse exame mais sensível para diagnosticar o estreitamento esofágico do que a própria endoscopia, direcionando assim o manejo adequado durante a EDA (MUIR AB, et al. 2016).

Uma metanálise recente demonstrou que, em pacientes com EoE, os IBP alcançam em mais de $50 \%$ dos pacientes remissão histológica (GUTIÉRREZ-JUNQUERA C, et al. 2018). Atualmente, é sugerida terapia com IBP $1 \mathrm{mg} / \mathrm{kg} /$ dose duas vezes ao dia em crianças, por um período de 8 a 12 semanas. Após esse tempo, deve-se realizar nova EDA e se a biópsias coletadas mostrarem redução abaixo de 15 eosinófilos/CGA, o IBP é mantido. Porém, se não houver resposta satisfatória, priorizam-se outras opções terapêuticas (GUTIÉRREZ-JUNQUERA C, et al. 2018).

Os corticosteroides sistêmicos são os mais prescritos no tratamento da EoE. Sua utilização deve ser considerada apenas para aliviar de forma imediata os sintomas do paciente com disfagia grave, desidratação bem como a perda de peso ou estenoses esofágicas. A dose para surtir efeito considerável é de $1 \mathrm{a} 2 \mathrm{mg} / \mathrm{kg} /$ dia de prednisona, com dose máxima de 40 a $60 \mathrm{mg}$ (CARR S, et al. 2018).

Os esteroides tópicos como propionato de fluticasona podem ser administrados após serem deglutidos em pulverização a partir de um inalador de medida de dose. A dosagem varia de 88 a $440 \mu \mathrm{g}$ duas a quatro vezes por dia para crianças (MEHTA P e FURUTA GT, 2015). A budesonida oral também pode ser usada, misturando o composto líquido da seguinte forma: budesonida $1 \mathrm{mg} / 2 \mathrm{ml}$ e $5 \mathrm{~g}$ de sucralose a fim de resultar em uma pasta retardando o trânsito luminal no esôfago. A dose indicada é de $1 \mathrm{mg}$ por dia para crianças com idade abaixo de 10 anos e $2 \mathrm{mg}$ ao dia para maiores de 10 anos (MEHTA P e FURUTA GT, 2015). O uso prolongado de corticosteroides aumenta as chances de reações adversas como aftas, esofagite fúngica por Candidae virais oportunistas. Existem outras preocupações no que tange ao uso prolongado que podem refletir na desmineralização óssea e insuficiência adrenal (GUPTA SK, 2014).

Admitindo-se os riscos de uso contínuo de corticoides sistêmicos, os corticoides tópicos foram analisados e demonstraram maior eficácia para atingir a resolução de lesões histológicas. Em contrapartida, um ensaio prospectivo pediátrico comparou a prednisona oral com fluticasona tópica deglutida demonstrando similaridade nas respostas inflamatórias assim como a mesma proporção da recidiva quando descontinuados (GUPTA SK, 2014).

Outros medicamentos como o cromoglicato de sódio ou o montelucaste, antagonistas dos receptores de leucotrienos, não são recomendados para o tratamento da EoE por falta de benefícios reais. O mesmo vale para imunossupressores e biológicos, que por mais que mostraram algum valor, o efeito é ainda limitado, não sendo terapia padrão (WECHLER JB, et al. 2014).

Os dois anticorpos monoclonais disponíveis contra IL-5, o mepolizumabe e o reslizumabe, têm sido utilizados para controlar a inflamação eosinofílica na EoE (MEHTA P e FURUTA GT, 2015). Ambos reduzem o número de eosinófilos nas biópsias esofágicas em adultos e crianças com EoE, entretanto, alguns estudos demonstraram que poucos pacientes conseguiram atingir números normais e a resposta clínica foi semelhante ao placebo (CARR S, et al. 2018). Outra modalidade terapêutica é a dilatação esofágica, utilizada em pacientes com estreitamento esofágico segmentar ou estenoses esofágicas focais, facilitando a passagem de alimentos (CARR S, et al. 2018). A complicação mais comum, embora de pouca incidência, é a dor no peito, podendo ocorrer outras mais raras como perfuração ou hemorragia (MEHTA P e FURUTA GT, 2015). 
O tratamento dietético é uma abordagem tão importante quanto as outras disponíveis. Como os pacientes apresentam uma resposta alérgica contra um antígeno (como os alimentos), é crucial a retirada de tais alimentos da dieta, promovendo uma resposta clínica e histológica da doença (CARR S, et al. 2018).

As três possíveis formas de abordagem alimentar são: dieta elementar, dieta empírica Carr S, et al. (2018) e dieta baseada nos resultados de testes de alergia (KAGALWALLA AF, 2014).

A dieta elementar é baseada na administração de uma fórmula líquida que contém nutrientes considerados essenciais para a alimentação destes indivíduos (CARR S, et al. 2018). Baseia-se na administração de uma fórmula constituída de aminoácidos. Esta conduta é reservada para pacientes que não conseguem estabilizar seu quadro com a dieta de eliminação e/ou quando o tratamento médico não tiver sido eficaz. As desvantagens estão relacionadas com o custo mais elevado e sabor desagradável (KAGALWALLA AF, 2014).

Considerando que o paciente apresentou boa resposta ao tratamento (tanto clínica quanto histológica), alimentos passam a ser inseridos aos poucos para estes indivíduos (CARR S, et al. 2018). Segundo a literatura, a introdução alimentar varia de 5 a 7 dias. Um mês após três ou quatro alimentos serem inseridos, é necessária a endoscopia para avaliar se o paciente apresentou tolerância aos mesmos (KAGALWALLA AF, 2014). A dieta empírica é baseada na eliminação de alimentos, sem testes de alergia prévios, que são considerados mais alergênicos de modo geral.

A dieta é baseada em uma alimentação com ausência de trigo, proteína do leite de vaca, soja, ovo, nozes, frutos do mar e amendoim. Dentre todos os alimentos citados anteriormente, o leite é o alérgeno desencadeador de doença mais comum. Ao excluir alimentos considerados fundamentais na dieta de uma criança, há uma chance de crescimento e desenvolvimento não adequado, levando a deficiências nutricionais (KAGALWALLA AF, 2014).

Além disso, deve-se evitar a contaminação cruzada de alimentos. A mesma acontece quando o alimento é processado, preparado, cozido ou servido. Para que a contaminação cruzada seja evitada deve-se ter uma lavagem adequada das mãos durante o preparado dos alimentos e utilização de recipientes diferentes (KAGALWALLA AF, 2014).

Outro tipo de abordagem está relacionado com a premissa de que como a esofagite eosinofílica está associada a uma resposta alérgica a um alimento ou aero alérgeno, poderiam ser realizados testes que poderiam detectar tais antígenos e os eliminar da dieta. Neste sentido, são usados os testes cutâneos por picada (SPT) ou prick-test e o teste de contato atópico (TCA) ou atopy patch test (APT) (WECHSLER JB, et al. 2014).

Alguns pacientes podem apresentar reações a diversos antígenos sendo muitas vezes não identificados nos testes de alergia. Outro fator seria a positividade para determinado alimento, mesmo que o alimento não seja o desencadeador da doença, retirando-o desnecessariamente da dieta (WECHLER JB, et al. 2014).

Portanto, a vantagem de escolha de tal abordagem é que uma quantidade menor de alimentos irão ser retirados da dieta do paciente. Além disto, extratos comerciais geram menos testes positivos que as preparações que utilizem alimentos frescos. Como já dito, a complexidade de tal patologia é uma das principais dificuldades da dieta baseada nos resultados de testes de alergia (WECHLER JB, et al. 2014).

\section{CONSIDERAÇÕES FINAIS}

A esofagite eosinofílica é uma doença inflamatória crônica, que deve ser considerada no diagnóstico diferencial de uma disfagia, essencialmente em pacientes sem evidência de DRGE. O quadro clínico apresenta sintomas variados conforme a faixa etária e os achados desta patologia, ainda que não patognomônicos, são formação anelar esofágica à EDA, fragilidade da mucosa com laceração fácil à passagem do endoscópio, edema, estenose e uma contagem superior a 15 eosinófilos por campo de grande ampliação. Dessa forma, sempre devem ser coletadas biopsias do esôfago mesmo que as áreas sejam visualmente normais. Dada a causa alérgica, a primeira escolha terapêutica deve ser a eliminação dietética dos alérgenos envolvidos, seguida pelo tratamento farmacológico se as queixas persistirem. 


\section{REFERÊNCIAS}

1. ACEVES SS. Remodeling and fibrosis in chronic eosinophil inflammation. DigDis. 2014; 32(1-2): p. 15-21.

2. BRAGA-TAVARES H, et al. Esofagite Eosinofílica: uma patologia emergente em Pediatria. Acta medica portuguesa. 2009; 22(5): p. 579-588.

3. BROWN-WHITEHORN TF, SPERGEL JM. The link between allergies and eosinophilic esophagitis: implications for management strategies. Expert RevClinImmunol. 2010 Jan; 6(1): p. 101

4. CARR S, et al. Eosinophilic esophagitis. Allergy Asthma Clin Immunol. 2018; 14(2): p. 58.

5. CHEGADE M. Em tempo: Esofagite eosinofílica: quando suspeitar e como diagnosticá-la em crianças e adolescentes. Rev Paul Pediatr. 2016; 34(4): p. 395-396.

6. CHENG E. Proton Pump Inhibitors for Eosinophilic Esophagitis. Curr Opin Gastroenterol. 2013 Jul; 29(4): p. 416420.

7. CIANFERONI A, et al. Recent advances in the pathological understanding of eosinophilic esophagitis. Expert Rev Gastroenterol Hepatol. 2015; 9(12): p. 1501-1510.

8. COUTO M, et al. Esofagite eosinofílica. Acta Med Port. 2011; 24(4): p. 949-960.

9. DURRANI S, ROTHENBERG M. Recent advances in eosinophilic esophagitis. F1000Res. 2017; 6: p. 1775.

10. GUITIÉRREZ-JUNQUERA C, et al. The Role of Proton Pump Inhibitors in the Management of Pediatric Eosinophilic Esophagitis. Front Pediatr. 2018 May; 6: p. 119.

11. GUPTA SK. Diagnostic and therapeutic challenges in pediatric eosinophilic esophagitis: past, present and future. Dig Dis. 2014; 32(1-2): p. 107-109.

12. HIRANO I, ACEVES SS. Clinical implications and pathogenesis of esophageal remodeling in eosinophilic esophagitis. Gastroenterol Clin North Am. 2014 Jun; 43(2): p. 297-316.

13. KAGALWALLA AF. Dietary treatment of eosinophilic esophagitis in children. DigDis. 2014; 32(1-2): p. 114-119.

14. LITOSH VA, et al. Calpain-14 and its association with eosinophilic esophagitis. J Allergy Clin Immunol. 2017 Jun; 139(6): p. 1762-1771.

15. LUCENDO AJ, et al. Guidelines on eosinophilic esophagitis: evidence-based statements and recommendations for diagnosis and management in children and adults. United European Gastroenterol J. 2017 Apr; 5(3): p. 335-358.

16. LYNCH MK, et al. Eosinophilic Esophagitis in Children: Implications for Health-Related Quality of Life and Potential Avenues for Future Research. J Pediatr Psychol. 2015 Sep; 40(8): p. 727-732.

17. MEHTA P, FURUTA GT. Eosinophils in Gastrointestinal Disorders: Eosinophilic Gastrointestinal Diseases, Celiac Disease, Inflammatory Bowel Diseases, and Parasitic Infections. Immunol Allergy Clin North Am. 2015 Aug; 35(5): p. 413-437.

18. MUIR AB, et al. Role of Endoscopy in Diagnosis and Management of Pediatric Eosinophilic Esophagitis. Gastrointest Endosc Clin N Am. 2016 Jan; 26(1): p. 187-200.

19. ROTHENBERG ME. Molecular, Genetic, and Cellular Bases for Treating Eosinophilic Esophagitis. Gastroenterology. 2015 May; 148(6): p. 1143-1157.

20. WECHSLER JB, BRYCE PJ. Allergic Mechanisms in Eosinophilic Esophagitis. GastroenterolClin North Am. 2014 June; 43(2): p. 281-296.

21. WECHSLER JB, et al. Elimination diets in the management of eosinophilic esophagitis. J Asthma Allergy. 2014; 7: p. 85-94. 